\title{
Ionization effect of solar particle GLE events in low and middle atmosphere
}

\author{
I. G. Usoskin ${ }^{1}$, G. A. Kovaltsov ${ }^{2}$, I. A. Mironova ${ }^{3}$, A. J. Tylka ${ }^{4}$, and W. F. Dietrich ${ }^{5}$ \\ ${ }^{1}$ Sodankylä Geophysical Observatory (Oulu unit), University of Oulu, Finland \\ ${ }^{2}$ Ioffe Physical-Technical Institute, St. Petersburg, Russia \\ ${ }^{3}$ Institute of Physics, St.Petersburg State University, St. Petersburg, Russia \\ ${ }^{4}$ Space Science Division, Naval Research Laboratory, Washington, DC, USA \\ ${ }^{5}$ Praxis, Inc., Alexandria, VA, USA
}

Received: 4 October 2010 - Published in Atmos. Chem. Phys. Discuss.: 14 December 2010

Revised: 17 February 2011 - Accepted: 18 February 2011 - Published: 3 March 2011

\begin{abstract}
Using a new reconstruction of the solar proton energy spectra for Ground Level Enhancement (GLE) events, based on fits to measurements from ground-based and satellite-borne instruments covering a wide energy range, we quantitatively evaluate the possible ionization effects in the low and middle atmosphere for 58 out of the 66 GLE events recorded by the world-wide neutron monitor network since 1956. The ionization computations are based on the numerical 3D CRAC:CRII model. A table of the ionization effect caused by the GLE events at different atmospheric heights is provided. It is shown that the direct ionization effect is negligible or even negative, due to the accompanying Forbush decreases, in all low- and mid-latitude regions. The ionization effect is important only in the polar atmosphere, where it can be dramatic in the middle and upper atmosphere (above $30 \mathrm{~km}$ ) during major GLE events.
\end{abstract}

\section{Introduction}

Cosmic rays form an important source of ionization of the Earth's atmosphere and the main source of the ionization in the troposphere and stratosphere. Most important for ionization in the troposphere-stratosphere are galactic cosmic rays (GCR) that possess high energies and produce a complicated cascade of secondary particles in the atmosphere, leading to permanent ionization of the ambient air. While the net energy brought by cosmic rays is small and the resulting ionization rate is not high, GCR can affect physical and chemical properties of the atmosphere. The process of cosmic ray

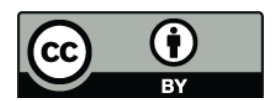

Correspondence to: I. G. Usoskin (ilya.usoskin@oulu.fi) induced ionization (CRII) in the lower atmosphere is well known and can be properly modelled using Monte-Carlo numerical models (see a review by Bazilevskaya et al., 2008). The upper atmosphere (above a few $\mathrm{g} / \mathrm{cm}^{2}$ ) is affected mostly by solar electromagnetic radiation and by lower energy particles of magnetospheric origin, which is also quite well modelled using straightforward analytical ionization models.

The GCR energy spectrum changes on different time scales, with the most apparent being a 11-year solar cycle, so that GCR flux is higher around solar minima. While the intensity of high energy GCR (above several tens of GeV) is rather stable, the lower energy part of the spectrum may change quite a bit - the difference in $100 \mathrm{MeV}$ particles flux can be an order of magnitude or more between the periods of solar minima and maxima. This variation is caused by the related changes in the heliospheric properties, such as polarity, strength and turbulence level of the interplanetary magnetic field, density and speed of solar wind, which are ultimately driven by the solar surface magnetic activity. This cyclic change in the GCR intensity is broadly called heliospheric modulation of cosmic rays and reaches $25 \%$ magnitude between maximum and minimum, as recorded by a ground-based polar neutron monitor (NM). The corresponding variations in CRII are also cyclic but their magnitude depends on the altitude and location (Bazilevskaya et al., 2008). On the other hand, solar-heliospheric transient phenomena, such as coronal mass ejections (CME), interplanetary shocks, corotating interaction regions, magnetic clouds may lead to strong but relatively short suppressions of the GCR intensity near Earth. Such suppressions are called Forbush decreases (Cane, 2000) which can be as strong as $25-30 \%$ in a polar NM count rate. The suppression itself develops quickly (within several hours up to one day), and the recovery may take days to weeks.

Published by Copernicus Publications on behalf of the European Geosciences Union. 
In addition to the permanently operating ionization process due to GCR, there are additional instantaneous atmospheric effects during relatively short periods of SEP (solar energetic particle) events, potentially affecting the Earth's environment (Miroshnichenko, 2008; Vainio et al., 2009). A typical SEP event is characterized by enhanced (sometimes by many orders of magnitude) flux of low energy $(<100$ $\mathrm{MeV}$ for protons) SEPs, which may last for several days (e.g., Cane et al., 1988; Reames et al., 1996; Lario and Simnett, 2004; Klecker et al., 2006). Because of low energy, such SEPs are not able to initiate atmospheric cascades and are stopped due to ionization losses; their ionization effect is limited to the upper polar atmosphere. This is usually modelled using analytical approximation of direct ionization (e.g., Vitt and Jackman, 1996). There are numerous studies of the effects caused by magnetospheric and solar energetic particles but they are usually limited to the upper atmosphere above $30 \mathrm{~km}$ (e.g., Jackman et al., 2008, 2009; Damiani et al., 2008; Seppälä et al., 2008). However, there is a special class of SEP events, called Ground Level Enhancement (GLE) events, which are characterized by higher energy of solar particles that can extend up to $\sim 1-10 \mathrm{GeV}$ (e.g., Duggal, 1979; Stoker, 1994). These energies are high enough to induce cascades of secondary particles in the atmosphere, just as GCRs do. Accordingly, an increase of the nucleonic component of the cascade can be measured at the ground level by NMs, which is a standard tool to record cosmic-ray intensities. Therefore, the ionizing atmospheric effect of GLE events is expected to be noticeable in the lower atmosphere. Phenomenological studies performed for the extremely large GLE event of 20 January 2005 suggest that an increase of ionization due to a severe SEP event is be significant in the polar low and middle atmosphere (Mironova et al., 2008). However, to the best of our knowledge, to date there have been no proper systematic computations of lower atmosphere ionization effects caused by GLE events. Estimates based on analytical or truncated numerical models of the atmospheric ionization that neglect development of the atmospheric cascade lead to potentially large errors in the lower-atmosphere ionization (Usoskin et al., 2010).

Here we straightforwardly compute, using the MonteCarlo CRAC:CRII model (Usoskin and Kovaltsov, 2006; Usoskin et al., 2004, 2010), ionization of lower and middle atmosphere during 58 out of the 66 GLE events of the last five solar cycles, using new reconstructions of the energy spectra of SEP protons (Tylka and Dietrich, 2009). (The remaining GLE events were too small or had too little data for spectral analysis.) We note that GLE events often occur on the background Forbush decrease, that can overcompensate the ionization enhancement due to SEPs, leading to the negative net atmospheric effect (i.e., reduced ionization), contrary to naive expectations. Here we systematically evaluate the combined ionization effect of both GCR and SEP in the low and middle atmosphere. We discuss in full detail the ionization effect of the GLE event of 20 January 2005, and for other events we briefly summarize the results. The result is primarily oriented to the atmospheric community and can be applied for evaluation of the middle atmosphere response to strong SEP events.

\section{Cosmic Ray Induced Ionization}

Nucleonic-muon-electromagnetic cascades initiated by energetic cosmic rays in the Earth's atmosphere lead to ionization of the ambient air at different altitudes. Generally the CRII rate (number of ion pairs produced in one gram of the ambient air per second) at a given residual atmospheric depth ${ }^{1} h$ can be represented as follows (Bazilevskaya et al., 2008):

$I\left(h, P_{c}, t\right)=\sum_{i} \int_{T_{c, i}}^{\infty} S_{i}(T, t) \cdot Y_{i}(h, T) d T$,

where the summation is performed over different $i$-th species of primary CR (protons, $\alpha$-particles, heavier nuclei), $Y_{i}(h, T)$ is the ionization yield function (the number of ion pairs produced at the atmospheric depth $h$ in the atmosphere by the unit flux of CR particles of the $i$-th type with kinetic energy $T), S_{i}(T, t)$ is the differential energy spectrum (in units of $\left[\mathrm{cm}^{2} \mathrm{sr} \mathrm{GeV} \mathrm{s}\right]^{-1}$ ) of galactic cosmic rays or solar energetic particles. The integration is performed above $T_{c, i}$, which is the kinetic energy of a particle of $i$-th type, corresponding to the local vertical geomagnetic cutoff rigidity $P_{c}$, which is the minimum rigidity (momentum per unit charge) that a charged particle must possess to overcome the shielding effect of the geomagnetic field and reach the given location (Cooke et al., 1991) in Earth's atmosphere. The value of $P_{c}$ varies from zero (in polar regions) to $15 \mathrm{GV}$ at equatorial regions. Full details of the CRII computations by the CRAC:CRII model, used here, are given by Usoskin and Kovaltsov (2006) and Usoskin et al. (2010). This model has been validated by comparisons with balloon-borne observations (Bazilevskaya et al., 2008) and with other models (e.g., Usoskin et al., 2009; Velinov et al., 2009; Atri et al., 2010). Note that CRII at a given location and time depends on three variables: altitude $h$ via the integrand yield function $Y$ (available in the tabular form in Usoskin and Kovaltsov (2006); Usoskin et al. (2010)), geographical location via the geomagnetic cutoff rigidity $P_{c}$ (integration limits), and time via the integrand GCR spectrum $S$. Since these three variables are mutually independent, they can be separated in order to solve

\footnotetext{
${ }^{1}$ Here we operate with the concept of residual atmospheric depth, which is the amount of matter (air) overburden above a given point in the atmosphere. The top of the atmosphere is $0 \mathrm{~g} / \mathrm{cm}^{2}$, and the mean sea-level corresponds to $h=1033 \mathrm{~g} / \mathrm{cm}^{2}$. This concept is naturally related to the development of the cascade and to the ionization. The atmospheric depth $h$ in $\left[\mathrm{g} / \mathrm{cm}^{2}\right]$ is linearly related to the static barometric pressure $p$ in $\mathrm{mb}$ (or hPa) as $h=1.0195 \cdot p$. Conversion to atmospheric height is not straightforward and depends on the exact vertical density profile.
} 
the problem numerically in an efficient way. The GCR spectrum is often approximated in the framework of the forcefield approach by fitting the measured data from the worldwide NM network (see Usoskin et al. (2005) for full details). In this framework, the GCR spectrum is parameterized via a single time-variable parameter, called the modulation potential $\phi$. The GCR-induced ionization rate was computed in this way for each of the days with GLE events analyzed here and also for the entire solar cycle 23 (1996-2008).

In order to compute the SEP-induced ion production during GLE events one needs to know the event-integrated energy spectrum of SEPs. Here we use event-integrated solar proton spectra derived by the method described in Tylka and Dietrich (2009); Tylka et al. (2011). The method begins by extracting the solar proton spectrum above $1 \mathrm{GV}$ rigidity (430 MeV kinetic energy) from the world-wide NM network using the NM yield function of Clem and Dorman (2000). Since a NM is sensitive to particles with energy above a few hundred $\mathrm{MeV} / \mathrm{nuc}$, it cannot provide data to reconstruct a lower energy part of the energy spectrum. Accordingly, satellite measurements were used in combination with NMbased results. For GLEs prior to 1973, proton fluences below $100 \mathrm{MeV}$ were taken from various catalogues (King, 1974; Reedy, 1977; Feynman and Gabriel, 1990), based on measurements from riometers and a few early satellites. Since 1973, the record of space-based solar proton measurements has been essentially continuous. For events between 1973 and 1986, the primary data source was the IMP8 satellite, which delivered proton measurements from three independent instruments that together covered the energy range of about 10-400 MeV. Having multiple instruments allowed for extensive cross-checks, including identification of unreliable channels and corrections for dead-time effects. After 1986 , integral proton fluences from $>10 \mathrm{MeV}$ to $>100 \mathrm{MeV}$ were provided by various GOES satellites. Careful comparisons between simultaneous IMP8 and GOES proton measurements (Feynman et al., 2000) has shown good agreement in the channels used in these GLE studies. For the 20 January 2005 and 13 December 2006 GLEs, additional proton measurements from SAMPEX and/or STEREO were also used. The validity of the analysis is confirmed by comparing the $\mathrm{NM}$ and satellite fluence measurements at nearly overlapping energies (Tylka and Dietrich, 2009) and by comparison with previously published studies of individual GLEs. Together the NM and satellite fluences are represented as an integral spectrum in rigidity. This combined integral spectrum is generally well fit to the Band functional form (Band et al., 1993), with point-to-point residuals on the order of $\sim 10 \%$ at satellite energies and of $\sim 30 \%$ at NM energies, which are relevant for the low and middle atmosphere. The Band function smoothly rolls one power-law into another, keeping both the function and its first derivative continuous. This Band function is a convenient starting point for atmospheric-ionization and other radiation-effect calculations, since it can be readily transformed into a differential spectrum in kinetic energy.
Let us express rigidity $R$ in GV and kinetic energy $T$ in $\mathrm{GeV}$. Then the integral omnidirectional event-integrated fluence (in protons $/ \mathrm{cm}^{2}$ ) of SEP is represented using the Band function:

$$
\begin{array}{ll}
J(>R)=J_{0} \cdot R^{-\gamma_{1}} \exp \left(-R / R_{0}\right), & \text { for } R \leq\left(\gamma_{2}-\gamma_{1}\right) R_{0}, \\
J(>R)=J_{0} \cdot A \cdot R^{-\gamma_{2}}, & \text { for } R>\left(\gamma_{2}-\gamma_{1}\right) R_{0},
\end{array}
$$

where

$$
\begin{aligned}
& A=\left[\left(\gamma_{2}-\gamma_{1}\right) R_{0}\right]^{\left(\gamma_{2}-\gamma_{1}\right)} \exp \left(\gamma_{1}-\gamma_{2}\right), \\
& R=\sqrt{T^{2}+2 T_{0} \cdot T}
\end{aligned}
$$

$T_{0}=0.938 \mathrm{GeV}$ is the proton's rest-mass energy. These equations correspond to the event-integrated differential spectrum in kinetic energy (in protons $/\left(\mathrm{cm}^{2} \mathrm{sr} \mathrm{GeV}\right)$ ):

$$
\begin{aligned}
& S=\frac{1}{4 \pi} J_{0} \cdot R^{-\gamma_{1}} \exp \left(-R / R_{0}\right) \frac{\left(\gamma_{1} R_{0}+R\right)\left(T+T_{0}\right)}{R^{2} R_{0}}, \quad \text { for } R \leq\left(\gamma_{2}-\gamma_{1}\right) R_{0}, \\
& S=\frac{1}{4 \pi} J_{0} \cdot A \cdot \gamma_{2} \cdot R^{-\gamma_{2}} \frac{T+T_{0}}{R^{2}}, \quad \text { for } R>\left(\gamma_{2}-\gamma_{1}\right) R_{0} .
\end{aligned}
$$

This function includes four parameters, $J_{0}, R_{0}, \gamma_{1}$ and $\gamma_{2}$, which were fitted from the data.

In this study we neglect $\alpha$-particles and heavier species of SEP, since their contribution is minor (Tylka et al., 1999; Tylka et al., 2006). However, heavier species were considered in full extent when calculating CRII from GCR where they play a role (Webber and Higbie, 2003; Usoskin and Kovaltsov, 2006). Since we are interested in the eventintegrated effect, we also average over the initial SEP anisotropy, which is typically large only for a comparatively short period of time in SEP events (e.g., Plainaki et al., 2007).

\section{SEP event of 20 January 2005}

In this section we discuss in detail the ionization effect of the GLE event of 20 January 2005. This event was the second strongest ever observed by the ground-based NMs with an increase exceeding 20-fold in 5-min data (54-fold in 1-min data) of the South Pole NM (Mewaldt, 2006; Plainaki et al., 2007; Belov et al., 2010). It was characterized by a shortlasting anisotropic component with a very hard spectrum followed by prolonged isotropic emission of SEPs with a softer spectrum (McCracken et al., 2008). The first anisotropic injection led to a strong but very short ionization pulse only in the South polar region but the event-integrated atmospheric ionization was fairly symmetric in both polar regions (Bütikofer et al., 2008).

The time profile of the polar Oulu NM count rate is shown in Fig. 1. Note that the relatively quiet first period, before 17 January 2005, was followed by a strong $15 \%$ Forbush decrease caused by an interplanetary disturbance (Papaioannou et al., 2010). The GLE of 20 January 2005 occurred at the 


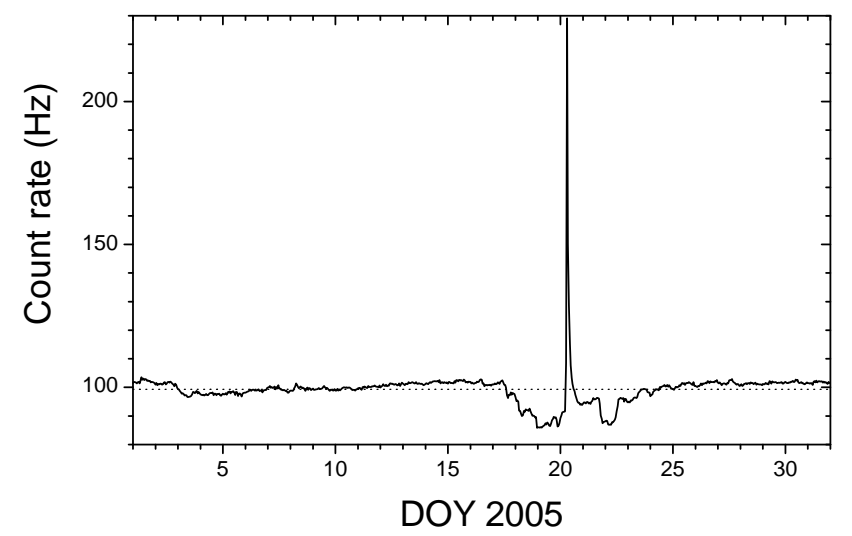

Fig. 1. Time profile of Oulu NM hourly averaged count rate for January 2005 (http://cosmicrays.oulu.fi). The dotted line represents the averaged monthly count rate for the month.

early recovery phase of the Forbush decrease when the background suppression of the NM count rate was about $10 \%$. During the following two days, an additional suppression of the CR intensity occurred, making the overall time profile even more complicated.

Atmospheric effects of this event have been studied by different groups (e.g. Bütikofer et al., 2008; Damiani et al., 2008; Seppälä et al., 2008; Mishev et al., 2010), but they were mostly concentrated on the upper polar atmosphere, discussed only the peak effect and neglected GCR variability. Here we are focused on the event-integrated ionization effect in the lower and middle atmosphere, with full consideration of the combined GCR+SEP variability.

The energy spectrum and intensity of cosmic rays varied quite a bit from day to day in January 2005. Figure 2a shows the SEP event-integrated spectrum computed using Eq. 4, along with the GCR proton fluence for the day of 20 January 2005 (including the effect of the Forbush decrease). The value of the modulation potential, computed from the NM network data using the approach of (Usoskin et al., 2005; Usoskin et al., 2011), for that day is $\phi_{\mathrm{d}}=1188 \mathrm{MV}$. The average GCR intensity for the whole month of January 2005 corresponded to the value of $\phi=788 \mathrm{MV}$. The corresponding average daily GCR proton fluence for January 2005 is also shown for comparison. One can see that for the day of 20 January SEPs heavily dominated below $1 \mathrm{GeV}$, but the effects quickly decreased with energy. On the other hand, the reduction of GCR fluence due to the Forbush decrease was significant in this energy range. Therefore, the CRII during January 2005 was an interplay between an enhancement due to SEPs and the reduction due to the Forbush decrease.

In order to study the ionization effect in full detail, we computed the daily averaged CRII rate in the polar region separately from SEPs and GCRs (Fig. 2b), using daily spectra shown in Fig. 2a. In all cases we assumed that the major ionization effect occurred within the $24 \mathrm{~h}$ following the onset of GLE. This assumption is valid for solar pro-
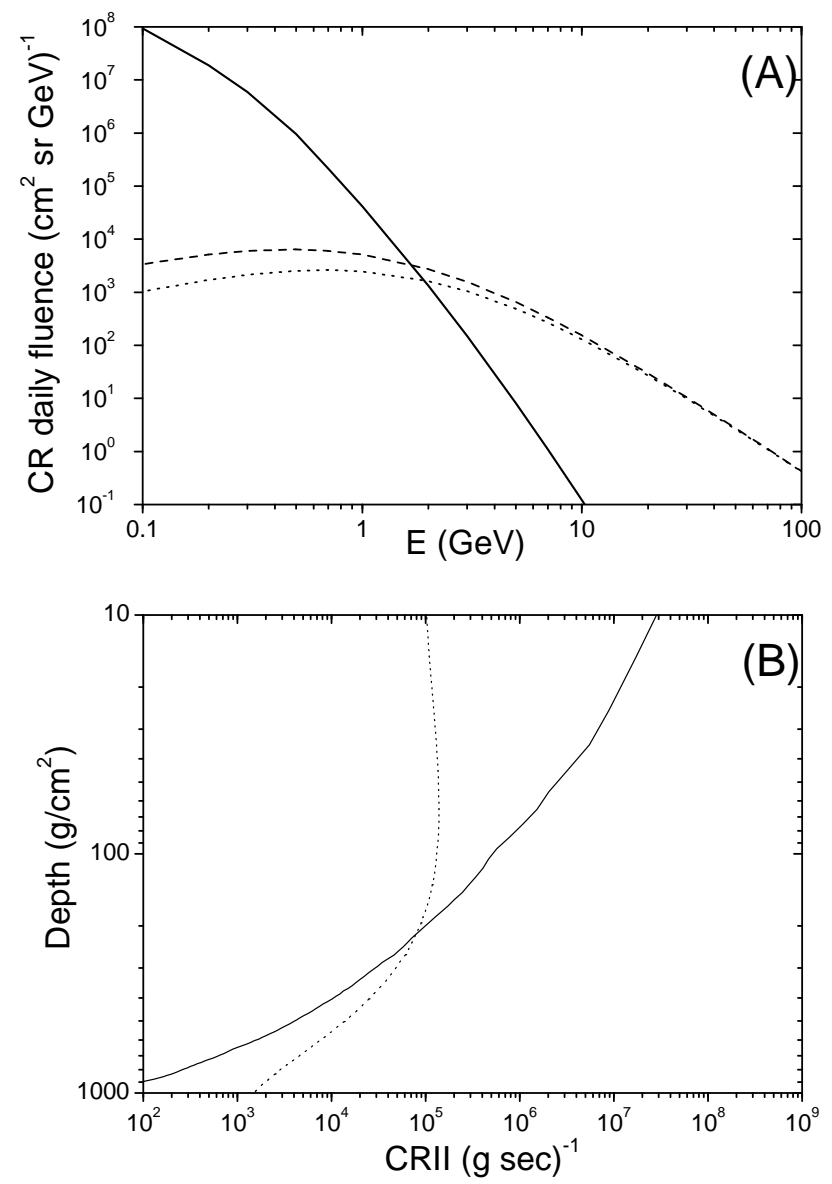

Fig. 2. Input proton spectra and the resulting atmospheric ionization for the event of 20 January 2005. (A) Differential fluence of solar protons from the 20 January 2005 event and the daily fluence of GCR protons for the day of 20 January 2005, including the effect of the Forbush decrease (dotted line). The dashed curve depicts the average GCR proton fluence for January 2005. (B) The vertical profile of the daily averaged CRII in the polar region from GCR (dotted curve) and SEP (solid curve) separately, for the day of 20 January 2005.

tons with energy above $100 \mathrm{MeV}$ and for ionization at the atmospheric depths larger than $100 \mathrm{~g} / \mathrm{cm}^{2}$ that are considered here; however, this assumption may lead to somewhat overestimated SEP ionization effect in the upper atmosphere for strongest SEP events, e.g., GLE \#42 (29 September 1989) and \#69 (20 January 2005). For GLEs like 19 October 1989 (\#43) and 4 November 2001 (\#62), with a strong secondary particle increase associated with the CME-driven shock's arrival at Earth, this secondary increase was not included in the ionization calculations. Consequently, effects in the upper atmosphere (at depths with less than $10 \mathrm{~g} / \mathrm{cm}^{2}$ ) are beyond the scope of this study. As one can see, e.g. in Fig. $2 \mathrm{~b}$, the SEP-induced ionization is significant above $h \approx 200 \mathrm{~g} / \mathrm{cm}^{2}$ (about $12 \mathrm{~km}$ altitude), but is subtle in the troposphere $\left(h \approx 500 \mathrm{~g} / \mathrm{cm}^{2}\right.$, or $5.5 \mathrm{~km}$ altitude). However, 


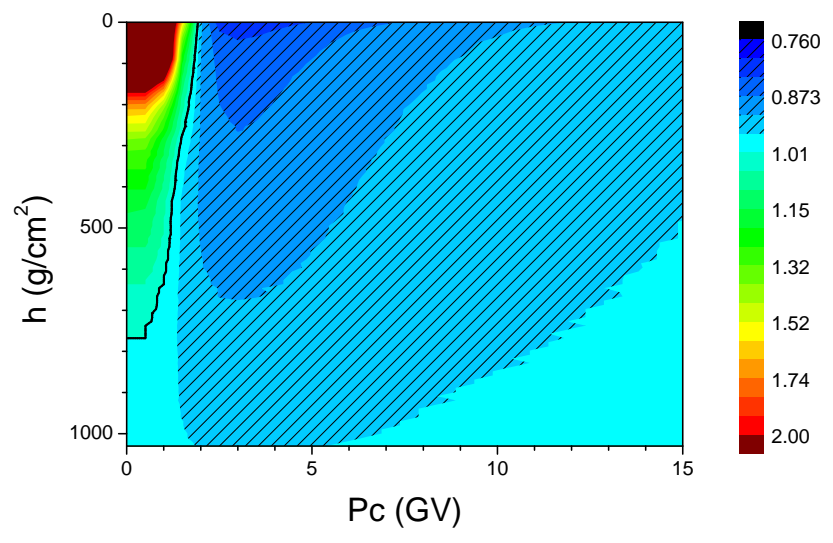

Fig. 3. The relative ionization effect $C$ (see text for definition) of GLE 20 January 2005 as function of the geomagnetic cutoff rigidity $P_{c}$ and atmospheric depth $h$. The region of suppression $(C<0.99)$ effect is hatched, while the solid curve bounds enhancement $(C>$ 1.01) effect.

these ionization rates quickly become smaller with decreasing geomagnetic latitude. The SEP ionization effect vanishes already at geomagnetic latitude of about $53^{\circ}\left(P_{c} \approx 2 \mathrm{GV}\right)$, even in the upper atmosphere.

Let us now define the absolute $C$ and relative $c$ CRII effect (at fixed altitude $h$ and location $P_{c}$ ) of a SEP event as follows:

$C\left(h, P_{c}\right)=\frac{I_{\mathrm{SEP}}+I_{\mathrm{GCR}}}{\langle I\rangle}, \quad c=(C-1) \cdot 100 \%$

where $I_{\mathrm{SEP}}$ and $I_{\mathrm{GCR}}$ are daily CRII production rates by SEP and GCR separately for the very day of event, and $\langle I\rangle$ is the averaged daily CRII for the whole month.

A 2-D (altitude vs. geomagnetic latitude) chart of the thus defined effect $C$ is shown in Fig. 3 for the event of 20 January 2005. The effect is a reduction (i.e. $C<1$ ) in the major part of the atmosphere because of the Forbush decrease of GCR. Less energetic SEPs are effectively rejected from lower altitudes and latitudes because of the atmosphere and geomagnetic cutoffs. Note that the overall effect is small in equatorial regions, being only a few percent. The increase $(C>1)$ is observed only in the polar upper atmosphere $\left(P_{c}<2 \mathrm{GV}\right.$, $h<700 \mathrm{~g} / \mathrm{cm}^{2}$ ).

In order to illustrate this, we have plotted in Fig. 4 the computed temporal variability of CRII (as the ionization effect $C$ ) during the month of January 2005 at two atmospheric depths, roughly corresponding to the tropopause $\left(h=200 \mathrm{~g} / \mathrm{cm}^{2}\right)$ and middle troposphere $\left(h=500 \mathrm{~g} / \mathrm{cm}^{2}\right)$, as a function of the local geomagnetic cutoff $P_{c}$. One can see the main feature a flattish profile before 17 January and after 25 January with a fractured dip during 17-24 January. The fracture (seeming increase) above $5 \mathrm{GV}$ cutoff in the middle of the dip was caused not by the GLE itself, but rather by the complicated CR intensity time profile (Fig. 1), when the recovery phase of the Forbush decrease was interrupted by another suppression during 21-22 January. We note that the GLE per se was

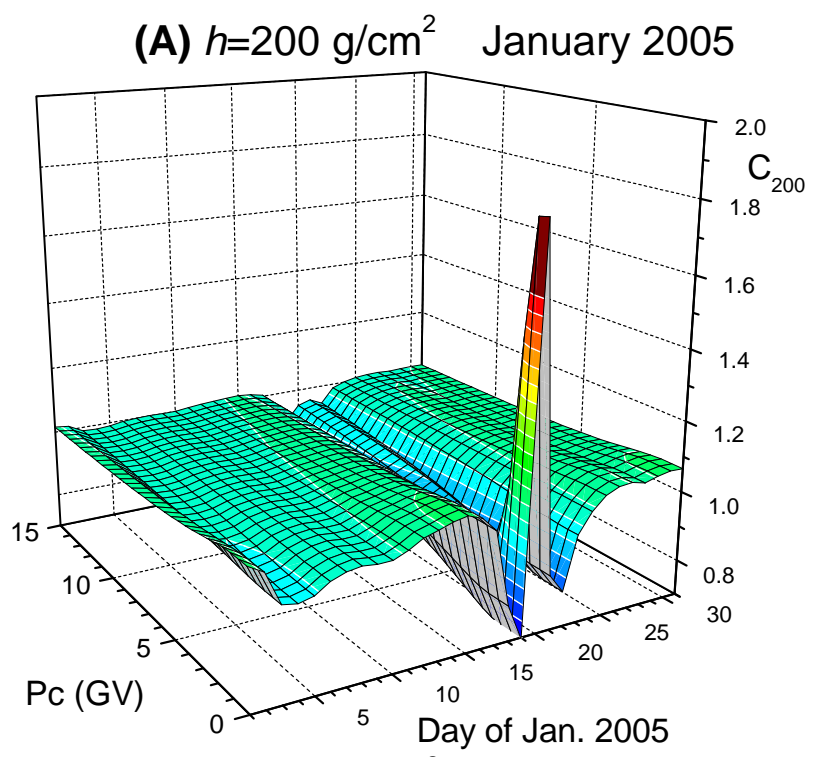

(B) $h=500 \mathrm{~g} / \mathrm{cm}^{2}$ January 2005

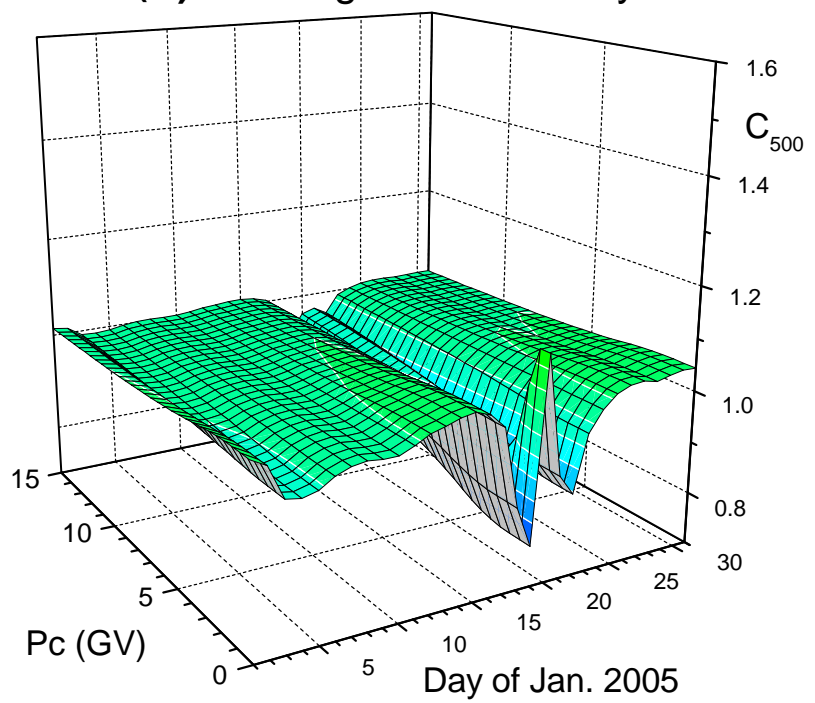

Fig. 4. The relative ionization $C$ (normalized to the average ionization rate in January, 2005) as function of time and location $\left(P_{c}\right)$ for two atmospheric depths, $200 \mathrm{~g} / \mathrm{cm}^{2}$ (panel A) and $500 \mathrm{~g} / \mathrm{cm}^{2}$ (panel B).

able to compensate the effect of the Forbush decrease only in the high-latitude region with $P_{c}<2 \mathrm{GV}$. Note that the enhancement of the daily ionization due to GLE was subtle in the polar troposphere but significant in the stratosphere and higher. Therefore, even for such a severe GLE, the ionization effect was negative and small in the major part of the atmosphere and positive only in the middle and upper polar atmosphere. 


\section{Results for other GLEs}

Here we summarize the results for all the GLE events for the last five solar cycles (1956-2006), for which it is possible to evaluate the SEP spectrum. We note that each GLE has an official number given by the International cosmic ray community (Shea et al., 1987), and presently there are 70 GLE events recorded since 1942 (see http://data.aad.gov.au/aadc/ gle/). GLE events \#1 through 4 were recorded by ion chambers before the NM era and no spectrum parametrization is possible. Thus, one analysis starts with GLE \# 5 (1956-Feb23) which was the strongest among all and covers the whole range until GLE \# 70 (2006-Dec-13). However, GLE \# 6, 14, $15,18,20,33$, and 34 were not considered, since they were too weak in NM count rates (Duggal, 1979; Stoker, 1994; Belov et al., 2010), to support reliable spectrum reconstruction. A weak GLE \# 16 and stronger GLE \#17 occurred on the same day and were merged together.

The events analyzed are listed in Table 1 together with the estimated effects at different altitudes in the polar atmosphere. Here we show the mean daily ionization due to SEPs only at the day of the GLE at two atmospheric depths, $100 \mathrm{~g} / \mathrm{cm}^{2}(\approx 16 \mathrm{~km}$ altitude $)$ and $300 \mathrm{~g} / \mathrm{cm}^{2}(\approx 9 \mathrm{~km})$, denoted as $I_{100}$ and $I_{300}$, respectively. We also show the relative ionization effect $c$ (Eq. 5) at four atmospheric levels of $100,300,500$ and $700 \mathrm{~g} / \mathrm{cm}^{2}$ corresponding to about $16 \mathrm{~km}$, $9 \mathrm{~km}, 5.5 \mathrm{~km}$, and $3 \mathrm{~km}$ altitudes, respectively. The Table is sorted according to the SEP-induced ionization in the lower stratosphere $I_{100}$.

\section{Discussion and conclusions}

One can see from Table 1 that the extreme event (the strongest GLE with a 90-fold increase at Leeds NM) of 23 February 1956 led (in the framework of the available data on SEP event-integrated spectrum) to a 33-fold increase of the daily ionization rate in the stratosphere and to a tripling in the lower troposphere. On the other hand, the second strongest GLE event (a 54-fold increase at South Pole NM) of 20 January 2005 is only number 7 in the strength of stratospheric ionization effect. As the strength of GLE we consider here the maximum increase of count rate among all ground-based NMs in the world network (see Table 1). The formal correlation between the GLE strength and the stratospheric effect $c_{100}$ in Table 1 is $r=0.83 \pm 0.05$, but it is totally defined by the two severe GLE events \#5 and 69 . However, the other 56 points depict wider scatter and poorer correlation $r=0.53 \pm 0.11$ (see Fig. 5). Although this correlation is statistically significant, the strength of the GLE event is not a particularly reliable indicator of the overall ionization effect of the event. This result is not unexpected, in that the peak count rate of a polar NM during GLE event is often caused by a highly anisotropic short impulsive phase of the event with a hard spectrum, while the main isotropic phase may have a

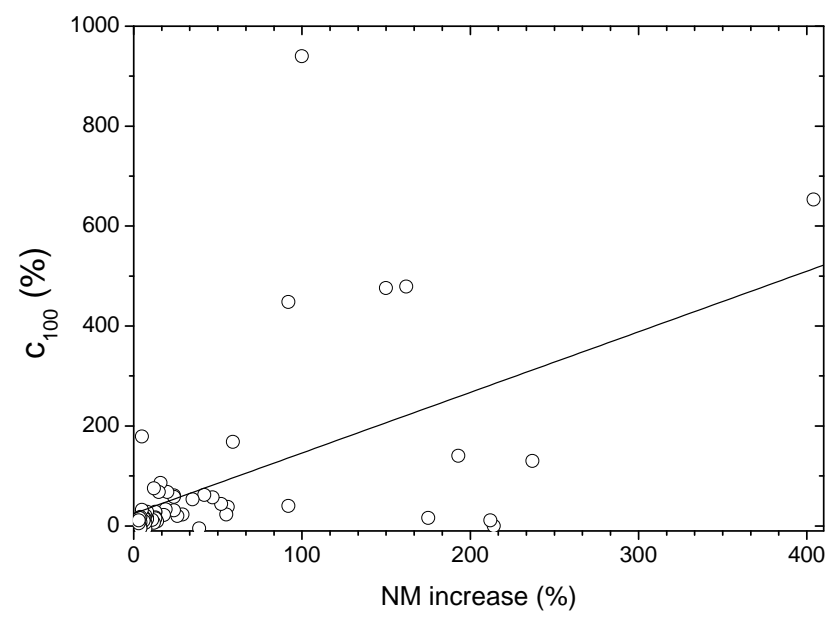

Fig. 5. Scatter plot of the relative CRII effect $c_{100}$ vs. the GLE strength (NM increase) - see Table 1 along with the best fit regression line. The two strongest GLE events \#5 (23 February 1956) and 69 (20 January 2005), whose NM increases are larger that these by more than an order of magnitude, are not included into the plot.

softer spectrum and lower intensity, as was in the case of the event of 20 January 2005. Thus, the use of the formal GLE strength as a proxy for the possible ionization effect in the polar atmosphere may be misleading.

A strong correlation $(r>0.97)$ exists between the SEPinduced ionization $I_{100}$ and the relative ionization effect $c_{100}$ in the stratosphere, even if the seven strongest events $\left(I_{100}>5 \cdot 10^{4} \mathrm{~g}^{-1} \mathrm{~s}^{-1}\right)$ are removed. There is no correlation between GLE strength and ionization in the lower troposphere - the overall correlation between $I_{100}$ and the effect in the low troposphere $c_{700}$ is strong enough $(r \approx 0.8)$ but it is totally defined by the strongest events. Removal of the seven strong events leads to a formally negative correlation $r=-0.25 \pm 0.12$. This indicates that the ionization of the troposphere is defined not by the additional SEP flux but rather by the variability of the GCR flux, in particular by the Forbush decrease. The range of variations of the daily GCRinduced ionization rate, within a month, varies between -32 and $10 \%,-25$ and $7 \%,-20$ and $5 \%$, and -15 and $4 \%$ for the atmospheric depths of $100,300,500$ and $700 \mathrm{~g} / \mathrm{cm}^{2}$, respectively. Accordingly, the ionization of the lower troposphere during a day of GLE is largely defined (except for a few extremely strong events) mostly by the GCR background.

As an example of interplay between GCR- and SEPinduced variability of CRII, we show on Fig. 6 temporal profile of the ionization rate in the polar region at two atmospheric depths, 100 and $300 \mathrm{~g} / \mathrm{cm}^{2}$, during the solar cycle 23 . A few spikes correspond to the GLE events (as denoted at the top panel), while the long-term variation is due to GCR modulation. The effect of SEP, visible at $h=100 \mathrm{~g} / \mathrm{cm}^{2}$, diminished already at $300 \mathrm{~g} / \mathrm{cm}^{2} \mathrm{~m}$ with only the event of 20 January 2005 producing a small observable increase. This is in general agreement with some earlier case studies, e.g., 
Table 1. Computed ionization effect of GLE events, ordered according to their ionization effect $I_{100}$ at $100 \mathrm{~g} / \mathrm{cm}^{2}$. The official GLE number, the date, and the strength (maximum increase in \% of NM count rate - see Duggal (1979); Belov et al. (2010)) of the events are given in the first three columns. Daily mean ionization due to SEP only at 100 and $300 \mathrm{~g} / \mathrm{cm}^{2}$ atmospheric depths is given in $10^{4}$ ( $\mathrm{g} \mathrm{s}$ ) ${ }^{-1}$ as $\mathrm{I}_{100}$ and $\mathrm{I}_{300}$, respectively. Relative CRII effect $c$ in \% (Eq. 5) at different depths $h$ is shown in columns \#6-9). All the values are given for the polar atmosphere.

\begin{tabular}{|c|c|c|c|c|c|c|c|c|}
\hline Date & GLE No. & NM increase & $\mathrm{I}_{100}$ & $\mathrm{I}_{300}$ & $\mathrm{c}_{100}$ & $c_{300}$ & $\mathrm{c}_{500}$ & $c_{700}$ \\
\hline 23 Feb 1956 & 5 & 9000 & 681 & 44.1 & 3370 & 680 & 365 & 196 \\
\hline $12 \operatorname{Nov} 1960$ & 10 & 100 & 135 & 7.26 & 940 & 140 & 67 & 31 \\
\hline 29 Sep 1989 & 42 & 404 & 84.7 & 5.81 & 653 & 125 & 64 & 34 \\
\hline 15 Nov 1960 & 11 & 150 & 71.8 & 3.8 & 476 & 58 & 22 & 7 \\
\hline 24 Oct 1989 & 45 & 162 & 60 & 3.47 & 479 & 67 & 30 & 12 \\
\hline 19 Oct 1989 & 43 & 92 & 59.9 & 3.16 & 448 & 68 & 31 & 14 \\
\hline 20 Jan 2005 & 69 & 5400 & 51.8 & 2.92 & 285 & 35 & 13 & 3 \\
\hline 17 Jul 1959 & 7 & 5 & 25.6 & 1.48 & 179 & 9 & -5 & -9 \\
\hline $14 \mathrm{Jul} 2000$ & 59 & 59 & 23.8 & 1.36 & 168 & 20 & 5 & 0 \\
\hline 15 Apr 2001 & 60 & 237 & 20.4 & 1.16 & 130 & 16 & 6 & 1 \\
\hline 22 Oct 1989 & 44 & 193 & 19.7 & 1.13 & 140 & 9 & -3 & -6 \\
\hline 01 Sep 1971 & 23 & 16 & 17.1 & 0.892 & 86 & 16 & 9 & 5 \\
\hline 04 Aug 1972 & 24 & 15 & 13.4 & 0.751 & 68 & 10 & 2 & 1 \\
\hline 28 Jan 1967 & $16+17$ & 20 & 13 & 0.761 & 68 & 12 & 6 & 3 \\
\hline 29 Oct 2003 & 66 & 35 & 12.8 & 0.71 & 53 & -11 & -14 & -13 \\
\hline 13 Dec 2006 & 70 & 92 & 10.2 & 0.588 & 40 & 4 & 1 & -4 \\
\hline $18 \mathrm{Jul} 1961$ & 13 & 24 & 9.97 & 0.575 & 61 & 7 & 2 & 0 \\
\hline 28 Oct 2003 & 65 & 47 & 9.67 & 0.557 & 57 & 8 & 2 & 0 \\
\hline 16 Aug 1989 & 41 & 24 & 8.69 & 0.504 & 58 & 6 & 1 & -4 \\
\hline 11 Jun 1991 & 51 & 12 & 8.63 & 0.527 & 75 & -1 & -6 & -7 \\
\hline 15 Jun 1991 & 52 & 42 & 7.75 & 0.442 & 62 & -5 & -9 & -9 \\
\hline 6 Nov 1997 & 55 & 19 & 7.11 & 0.426 & 33 & 7 & 4 & 2 \\
\hline 24 May 1990 & 48 & 52 & 5.59 & 0.352 & 44 & 5 & 1 & 0 \\
\hline 22 Nov 1977 & 30 & 55 & 5.28 & 0.311 & 23 & 4 & 2 & 1 \\
\hline 24 Jan 1971 & 22 & 29 & 5.12 & 0.287 & 23 & 1 & -1 & -1 \\
\hline 7 Aug 1972 & 25 & 8 & 4.96 & 0.281 & 16 & -3 & -4 & -3 \\
\hline 30 Mar 1969 & 21 & 5 & 4.83 & 0.337 & 32 & 8 & 5 & 1 \\
\hline 23 Sep 1978 & 32 & 13 & 4.79 & 0.281 & 27 & 7 & 4 & 3 \\
\hline 21 May 1990 & 47 & 24 & 4.23 & 0.296 & 31 & 2 & 0 & -1 \\
\hline 20 Nov 1960 & 12 & 7 & 4.2 & 0.255 & 21 & -1 & -2 & -2 \\
\hline 24 Sep 1977 & 29 & 11 & 4.06 & 0.24 & 12 & -1 & -2 & -2 \\
\hline 26 May 1990 & 49 & 13 & 3.66 & 0.208 & 28 & 2 & 0 & -1 \\
\hline 8 Dec 1982 & 38 & 56 & 3.62 & 0.231 & 38 & 12 & 8 & 5 \\
\hline 2 Nov 1992 & 54 & 6.5 & 3.57 & 0.206 & 16 & 1 & 0 & -1 \\
\hline 4 Nov 2001 & 62 & 8 & 3.32 & 0.191 & 28 & 8 & 5 & 3 \\
\hline 12 Oct 1981 & 36 & 18 & 3.04 & 0.186 & 22 & 4 & 2 & 1 \\
\hline 18 Apr 2001 & 61 & 26 & 2.82 & 0.175 & 20 & 4 & 2 & 1 \\
\hline 3 Sep 1960 & 9 & 4 & 2.76 & 0.163 & 18 & 3 & 1 & 0 \\
\hline 4 May 1960 & 8 & 175 & 2.71 & 0.201 & 16 & 2 & 0 & 0 \\
\hline 18 Nov 1968 & 19 & 13 & 2.56 & 0.142 & 17 & 2 & 1 & 0 \\
\hline 28 May 1990 & 50 & 6 & 2.3 & 0.144 & 16 & 0 & -1 & -1 \\
\hline 17 Jan 2005 & 68 & 3.5 & 2.29 & 0.133 & 16 & 4 & 2 & 1 \\
\hline 2 Nov 2003 & 67 & 39 & 2.22 & 0.122 & -5 & -13 & -12 & -9 \\
\hline 26 Dec 2001 & 63 & 13 & 1.89 & 0.103 & 15 & 4 & 3 & 2 \\
\hline 7 May 1978 & 31 & 214 & 1.65 & 0.128 & 0 & -4 & -3 & -3 \\
\hline 16 Feb 1984 & 39 & 212 & 1.53 & 0.0949 & 11 & 3 & 2 & 1 \\
\hline 26 Nov 1982 & 37 & 6 & 1.32 & 0.084 & 10 & 2 & 1 & 0 \\
\hline 24 Aug 2002 & 64 & 14 & 1.29 & 0.0714 & 9 & 2 & 1 & 0 \\
\hline 30 Apr 1976 & 27 & 4 & 1.21 & 0.0677 & 7 & 2 & 1 & 1 \\
\hline 19 Sep 1977 & 28 & 3 & 1.19 & 0.063 & 5 & 0 & 0 & 0 \\
\hline 2 May 1998 & 56 & 7 & 1.08 & 0.0592 & -6 & -7 & -6 & -4 \\
\hline
\end{tabular}


Table 1. Continued.

\begin{tabular}{lcr|rc|rrrr}
\hline Date & GLE No. & NM increase & $\mathrm{I}_{100}$ & $\mathrm{I}_{300}$ & $\mathrm{c}_{100}$ & $\mathrm{c}_{300}$ & $\mathrm{c}_{500}$ & $\mathrm{c}_{700}$ \\
\hline 25 Jun 1992 & 53 & 7 & 1 & 0.0562 & 6 & 1 & 0 & 0 \\
25 Jul 1989 & 40 & 8 & 0.988 & 0.0527 & 13 & 5 & 4 & 3 \\
24 Aug 1998 & 58 & 4 & 0.889 & 0.0549 & -2 & -3 & -3 & -2 \\
15 Nov 1989 & 46 & 12 & 0.756 & 0.0438 & 6 & 0 & 0 & 0 \\
29 Apr 1973 & 26 & 3 & 0.649 & 0.0442 & 5 & 2 & 1 & 1 \\
10 May 1981 & 35 & 3 & 0.562 & 0.0317 & 11 & 5 & 4 & 3 \\
6 May 1998 & 57 & 4 & 0.243 & 0.0131 & -5 & -4 & -3 & -2 \\
\hline
\end{tabular}

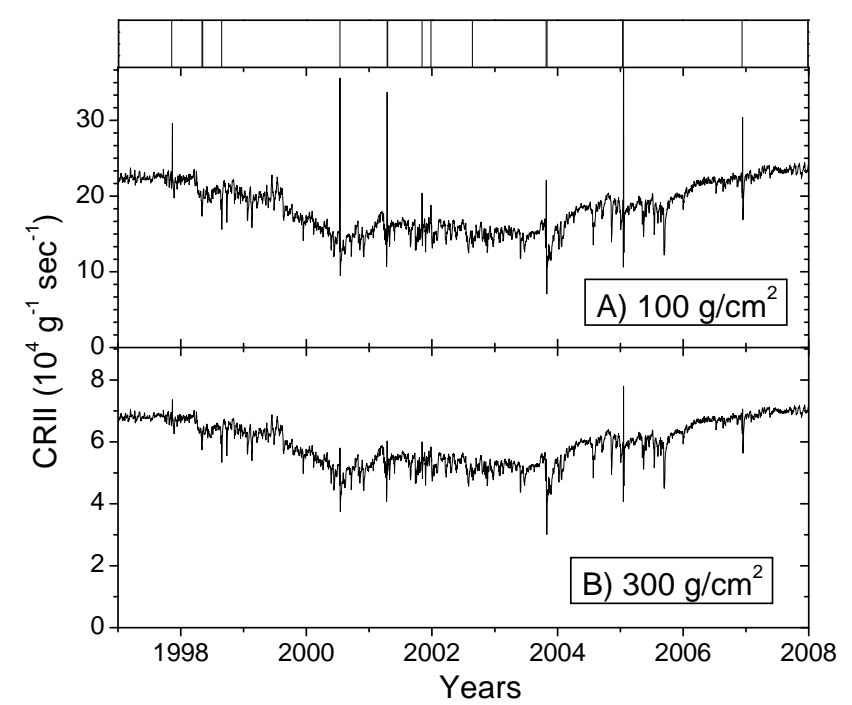

Fig. 6. Daily cosmic ray induced ionization at two atmospheric layers, $100 \mathrm{~g} / \mathrm{cm}^{2}$ (panel A) and $300 \mathrm{~g} / \mathrm{cm}^{2}$ (panel B), during the solar cycle \# 23. Note that the CRII value for the day of 20 January 2005 goes far beyond the Y-scale of panel A. Dates of the GLE events \#55 through 70 (see Table 1) are indicated in the top panel.

October-November 2003 (Wissing and Kallenrode, 2009; Jackman et al., 2005).

In conclusion, we have calculated the atmospheric ionization effect from nearly all of the GLE events since 1956. The results are presented in Table 1 for the polar atmosphere. There is no ionization effect at mid- or low-latitudes, even for the strongest events. We show that there is no straightforward relation between the strength of GLE (as measured by neutron monitors) and the ionization effect in polar atmosphere. The net atmospheric ionization effect is defined by an interplay between the SEP event itself and a Forbush decrease, which often accompanying it. This interplay makes it difficult to utilize regression or superposed epoch analysis in statistical studies of these effects. Accordingly, the atmospheric effect of SEP events should be studied individually, based on detailed information of the exact solar, heliospheric, and geospace conditions around the event.
Acknowledgement. Support from the Academy of Finland is acknowledged. GAK was partly supported by the Program of Presidium RAS N16-3-5.4. AJT and WFD were supported by the NASA Living with A Star Targeted Research and Technology Program, under DPR NNH09AL11I. IM acknowledges grant 11.37.26.2011 of St. Petersburg State University. This work was performed in relation with the international Programmes CAWSES-II and ISSI.

Edited by: M. Kulmala

\section{References}

Atri, D., Melott, A. L., and Thomas, B. C.: Lookup tables to compute high energy cosmic ray induced atmospheric ionization and changes in atmospheric chemistry, J. Cosmol. Astropart. Phys., 5, 008, doi:10.1088/1475-7516/2010/05/008, 2010.

Band, D., Matteson, J., Ford, L., Schaefer, B., Palmer, D., Teegarden, B., Cline, T., Briggs, M., Paciesas, W., Pendleton, G., Fishman, G., Kouveliotou, C., Meegan, C., Wilson, R., and Lestrade, P.: BATSE observations of gamma-ray burst spectra. I - Spectral diversity, Astrophys. J., 413, 281-292, doi:10.1086/172995, 1993.

Bazilevskaya, G. A., Usoskin, I. G., Flückiger, E. O., Harrison, R. G., Desorgher, L., Bütikofer, R., Krainev, M. B., Makhmutov, V. S., Stozhkov, Y. I., Svirzhevskaya, A. K., Svirzhevsky, N. S., and Kovaltsov, G. A.: Cosmic ray induced ion production in the atmosphere, Space Sci. Rev., 137, 149-173, doi:10.1007/s11214-008-9339-y, 2008.

Belov, A. V., Eroshenko, E. A., Kryakunova, O. N., Kurt, V. G., and Yanke, V. G.: Ground level enhancements of solar cosmic rays during the last three solar cycles, Geomag. Aeronom., 50, 21-33, doi:10.1134/S0016793210010032, 2010.

Bütikofer, R., Flückiger, E., Desorgher, L., and Moser, M.: The extreme solar cosmic ray particle event on 20 January 2005 and its influence on the radiation dose rate at aircraft altitude, Sci. Total Environ., 391, 177-183, 2008.

Cane, H. V.: Coronal Mass Ejections and Forbush Decreases, Space Sci. Rev., 93, 55-77, doi:10.1023/A:1026532125747, 2000.

Cane, H. V., Reames, D. V., and von Rosenvinge, T. T.: The role of interplanetary shocks in the longitude distribution of solar energetic particles, J. Geophys. Res., 93, 9555-9567, doi:10.1029/JA093iA09p09555, 1988.

Clem, J. and Dorman, L.: Neutron Monitor Response Functions, Space Sci. Rev., 93, 335-359, doi:10.1023/A:1026508915269, 
2000.

Cooke, D., Humble, J., Shea, M., Smart, D., Lund, N., Rasmussen, I., Byrnak, B., Goret, P., and Petrou, N.: On cosmic-ray cut-off terminology, Nuovo Cimento C, 14, 213-234, 1991.

Damiani, A., Storini, M., Laurenza, M., and Rafanelli, C.: Solar particle effects on minor components of the Polar atmosphere, Annales Geophys., 26, 361-370, 2008.

Duggal, S. P.: Relativistic solar cosmic rays, Rev. Geophys. Space Phys., 17, 1021-1058, 1979.

Feynman, J. and Gabriel, S.: Period and phase of the 88-year solar cycle and the Maunder minimum - Evidence for a chaotic sun, Solar Phys., 127, 393-403, 1990.

Feynman, J., Tylka, A., Reames, D., and Gabriel, S.: Near-Sun Energetic Particle Environment of Solar Probe-Phase 1, Tech. rep., JPL, 2000.

Jackman, C. H., DeLand, M. T., Labow, G. J., Fleming, E. L., Weisenstein, D. K., Ko, M. K. W., Sinnhuber, M., and Russell, J. M.: Neutral atmospheric influences of the solar proton events in October-November 2003, J. Geophys. Res., 110, A09S27, doi:10.1029/2004JA010888, 2005.

Jackman, C. H., Marsh, D. R., Vitt, F. M., Garcia, R. R., Fleming, E. L., Labow, G. J., Randall, C. E., López-Puertas, M., Funke, B., von Clarmann, T., and Stiller, G. P.: Short- and medium-term atmospheric constituent effects of very large solar proton events, Atmos. Chem. Phys., 8, 765-785, doi:10.5194/acp-8-765-2008, 2008.

Jackman, C. H., Marsh, D. R., Vitt, F. M., Garcia, R. R., Randall, C. E., Fleming, E. L., and Frith, S. M.: Long-term middle atmospheric influence of very large solar proton events, J. Geophys. Res., 114, D11304, doi:10.1029/2008JD011415, 2009.

King, J. H.: Solar Proton Fluences for 1977-1983 Space Missions, J. Spacecraft and Rockets, 11, 401-408, 1974.

Klecker, B., Kunow, H., Cane, H., Dalla, S., Heber, B., Kecskemety, K., Klein, K.-L., Kota, J., Kucharek, H., Lario, D., Lee, M., Popecki, M., Posner, A., Rodriguez-Pacheco, J., Sanderson, T., Simnett, G., and Roelof, E.: Energetic Particle Observations, Space Sci. Rev., 123, 217-250, 2006.

Lario, D. and Simnett, G. M.: Solar Energetic Particle Variations, in: Solar Variability and its Effects on Climate, edited by J. M. Pap, P. Fox, C. Frohlich, H. S. Hudson, J. Kuhn, J. McCormack, G. North, W. Sprigg, \& S. T. Wu, vol. 141 of AGU Geophysical Monograph Series, 195-216, Washington DC, USA, 2004.

McCracken, K. G., Moraal, H., and Stoker, P. H.: Investigation of the multiple-component structure of the 20 January 2005 cosmic ray ground level enhancement, J. Geophys. Res., 113, A12101, doi:10.1029/2007JA012829, 2008.

Mewaldt, R.: Solar Energetic Particle Composition, Energy Spectra, and Space Weather, Space Sci. Rev., 124, 303-316, doi:10.1007/s11214-006-9091-0, 2006.

Mironova, I. A., Desorgher, L., Usoskin, I. G., Flückiger, E. O., and Bütikofer, R.: Variations of aerosol optical properties during the extreme solar event in January 2005, Geophys. Res. Lett., 35, L18610, doi:10.1029/2008GL035120, 2008.

Miroshnichenko, L. I.: Solar cosmic rays in the system of solar terrestrial relations, J. Atmos. Solar-Terr. Phys., 70, 450-466, doi:10.1016/j.jastp.2007.08.027, 2008.

Mishev, A., Velinov, P., and Mateev, L.: Atmospheric Ionization Due to Solar Cosmic Rays from 20 January 2005 Calculated with
Monte Carlo Simulations, Compt. Rend. Acad. Bulgar Sci., 63, 1635-1642, 2010.

Papaioannou, A., Malandraki, O., Belov, A., Skoug, R., Mavromichalaki, H., Eroshenko, E., Abunin, A., and Lepri, S.: On the Analysis of the Complex Forbush Decreases of January 2005, Solar Phys., 266, 181-193, doi:10.1007/s11207-0109601-9, 2010.

Plainaki, C., Belov, A., Eroshenko, E., Mavromichalaki, H., and Yanke, V.: Modeling ground level enhancements: Event of 20 January 2005, J. Geophys. Res., 112, A04102, doi:10.1029/2006JA011926, 2007.

Reames, D. V., Barbier, L. M., and Ng, C. K.: The Spatial Distribution of Particles Accelerated by Coronal Mass Ejection-driven Shocks, Astrophys. J., 466, 473-486, doi:10.1086/177525, 1996.

Reedy, R.: Solar proton fluxes since 1956, in: Lunar and Planetary Science VIII, edited by Merril, R., Lunar and Planetary Institute, Houston, USA, 825-839, 1977.

Seppälä, A., Clilverd, M. A., Rodger, C. J., Verronen, P. T., and Turunen, E.: The effects of hard-spectra solar proton events on the middle atmosphere, J. Geophys. Res., 113, A11311, doi:10.1029/2008JA013517, 2008.

Shea, M. A., Smart, D. F., Humble, J. E., Fluckiger, E. O., Gentile, L. C., and Nichol: A Revised Standard Format for Cosmic Ray Ground-Level Event Data, in: Proc. 20th Internat. Cosmic Ray Conf., Moscow, USSR, 3, 171-174, 1987.

Stoker, P. H.: Relativistic Solar Proton Events, Space Sci. Rev., 73, 327-385, doi:10.1007/BF00751240, 1994.

Tylka, A. and Dietrich, W.: A new and comprehensive analysis of proton spectra in ground-level encahnced (GLE) solar particle events, in: 31th International Cosmic Ray Conference, Universal Academy Press, Lodź, Poland, 2009.

Tylka, A., Dietrich, W., Lopate, W., and Reames, D.: High-energy solar Fe in the 29 September 1989 Ground Level Event, in: 26th International Cosmic Ray Conference, edited by: Kieda, D., Salamon, M., and Dingus, B., Salt Lake City, Utah, USA, 6, 67-70, 1999.

Tylka, A. J., Cohen, C. M. S., Dietrich, W. F., Lee, M. A., Maclennan, C. G., Mewaldt, R. A., Ng, C. K., and Reames, D. V.: A Comparative Study of Ion Characteristics in the Large Gradual Solar Energetic Particle Events of 2002 April 21 and 2002 August 24, Astrophys. J. Suppl., 164, 536-551, doi:10.1086/503203, 2006.

Tylka, A. J., Dietrich, W. F., and Atwell, W.: Band function representation of solar proton spectra in Ground-Level Events, in preparation, 2011.

Usoskin, I. G. and Kovaltsov, G. A.: Cosmic ray induced ionization in the atmosphere: Full modeling and practical applications, J. Geophys. Res., 111, D21206, doi:10.1029/2006JD007150, 2006.

Usoskin, I. G., Gladysheva, O. G., and Kovaltsov, G. A.: Cosmic ray-induced ionization in the atmosphere: spatial and temporal changes, J. Atmos. Solar-Terr. Phys., 66, 1791-1796, doi:10.1016/j.jastp.2004.07.037, 2004.

Usoskin, I. G., Alanko-Huotari, K., Kovaltsov, G. A., and Mursula, K.: Heliospheric modulation of cosmic rays: Monthly reconstruction for 1951-2004, J. Geophys. Res., 110, A12108, doi:10.1029/2005JA011250, 2005.

Usoskin, I. G., Desorgher, L., Velinov, P., Storini, M., Flückiger, E. O., Bütikofer, R., and Kovaltsov, G. A.: Ionization of the earth's atmosphere by solar and galactic cosmic rays, Acta Geo- 
phys., 57, 88-101, doi:10.2478/s11600-008-0019-9, 2009.

Usoskin, I. G., Kovaltsov, G. A., and Mironova, I. A.: Cosmic ray induced ionization model CRAC:CRII: An extension to the upper atmosphere, J. Geophys. Res., 115, D10302, doi:10.1029/2009JD013142, 2010.

Usoskin, I. G., Kovaltsov, G. A., and Bazilevskaya, G. A.: Solar modulation parameter for cosmic rays since 1936 reconstructed from ground-based neutron monitors and ionization chambers, J. Geophys. Res., 116, A02104, doi:10.1029/2010JA016105, 2011.

Vainio, R., Desorgher, L., Heynderickx, D., Storini, M., Flückiger, E., Horne, R. B., Kovaltsov, G. A., Kudela, K., Laurenza, M., McKenna-Lawlor, S., Rothkaehl, H., and Usoskin, I. G.: Dynamics of the Earth's particle radiation environment, Space Sci. Rev., 147, 187-231, doi:10.1007/s11214-009-9496-7, 2009.

Velinov, P. I. Y., Mishev, A., and Mateev, L.: Model for induced ionization by galactic cosmic rays in the Earth atmosphere and ionosphere, Adv. Space Res., 44, 1002-1007, doi:10.1016/j.asr.2009.06.006, 2009.
Vitt, F. M. and Jackman, C. H.: A comparison of sources of odd nitrogen production from 1974 through 1993 in the Earth's middle atmosphere as calculated using a two-dimensional model, J. Reophys. Res., 101, 6729-6740, doi:10.1029/95JD03386, 1996.

Webber, W. and Higbie, P.: Production of cosmogenic Be nuclei in the Earth's atmosphere by cosmic rays: Its dependence on solar modulation and the interstellar cosmic ray spectrum, J. Geophys. Res., 108, 1355, doi:10.1029/2003JA009863, 2003.

Wissing, J. M. and Kallenrode, M.-B.: Atmospheric Ionization Module Osnabrück (AIMOS): A 3-D model to determine atmospheric ionization by energetic charged particles from different populations, J. Geophys. Res., 114, A06104, doi:10.1029/2008JA013884, 2009. 